\title{
Exposure assessment: the influence of environmental monitoring methodology
}

\author{
S. Viegas ${ }^{1,2}$, J. Prista $^{2}$ \& M. Gomes ${ }^{1,3}$ \\ ${ }^{1}$ Escola Superior de Tecnologia da Saúde de Lisboa-ESTeSL/IPL \\ (Higher School of Health Technologies of Lisbon - Polytechnic Institute \\ of Lisbon), Portugal \\ ${ }^{2}$ CIESP, Centro de Investigação e Estudos em Saúde Pública, \\ Escola Nacional de Saúde Pública - ENSP/UNL, \\ (National School of Public Health - New University of Lisbon), Portugal \\ ${ }^{3}$ REQUIMTE/CQFB, Chemistry Department, FCT, \\ New University of Lisbon, Portugal
}

\begin{abstract}
Exposure assessment is an important step of risk assessment process and has evolved more quickly than perhaps any aspect of the four-step risk paradigm (hazard identification, exposure assessment, dose-response analysis, and risk characterization). Nevertheless, some epidemiological studies have associated adverse health effects to a chemical exposure with an inadequate or absent exposure quantification.

In addition to the metric used, the truly representation of exposure by measurements depends on: the strategy of sampling, random collection of measurements, and similarity between the measured and unmeasured exposure groups.

Two environmental monitoring methodologies for formaldehyde occupational exposure were used to assess the influence of metric selection in exposure assessment and, consequently, in risk assessment process. In one of the methodologies, environmental samples were obtained by personal air sampling, and formaldehyde levels were measured by GC analysis and time-weighted average $\left(\mathrm{TWA}_{8}\right)$ estimated according to the NIOSH 2541 method. The second methodology aimed to measure ceiling values of formaldehyde using Photo Ionisation Detection equipment with simultaneously video recording. The NIOSH method data showed that exposure can be considered low, while results
\end{abstract}


obtained through Photo Ionisation Detection equipment show that majority of the cases are above the limits for TLV-Ceiling. This is of much more interest since formaldehyde effects are more related with peak concentrations than with average ones.

We can conclude that methodology to perform exposure assessment is crucial to risk assessment, and the metric and sampling strategy should consider the chemical toxicological mechanism.

Keywords: exposure assessment, environmental monitoring methodology, risk assessment, formaldehyde exposure.

\section{Introduction}

Occupational chemical exposure can be defined as the contact of any part of the human body with one or more chemical agents present in the workplace environment. This contact may occur mainly by inhalation, but also via dermal contact and even, inadvertently, by oral ingestion.

Exposure assessment, as a part of risk assessment process, evolved more rapidly than any other aspect of the four-step risk paradigm (hazard identification, exposure assessment, dose-response analysis, and risk characterization). Area measurements have been replaced by studies of microenvironments in which humans are likely to be exposed [1]. Therefore, studies need to be performed and analyzed in a way that is suitable for risk assessment. Information must be related with the dose-effect correlation and on whether there is evidence of a threshold effect [2].

Exposure can be assessed by representative monitoring data but it is always important to decide what and when to assess, and ensure measurements representativeness and reliability [3]. Usually, exposure monitoring requires measurement of chemical levels in workplace environment, since the main route of exposure is inhalation.

Moreover, it is crucial to get information concerning exposure conditions, and also toxicokinetic characteristics such as limited versus repeated exposures, dose-rate considerations, reversibility of toxicological process and composition of the exposed population [4].

Exposure assessment includes 5 basic steps: data collection, hazard identification, exposure group's formation, appropriate exposure metrics and exposures estimation selection [5].

Collected data include toxicological information on adverse health effects, tasks description and other information useful for sampling strategy. Identification of hazard requires careful study about the workplace and physical and toxicological principles to ensure a comprehensive evaluation. Exposure groups' formation is an essential component to the exposure assessment process, because it can simplify the process. However, the exposure groups should follow some requirements, namely work and environmental similarity, i.e., workers must have the same general exposure profile $[5,6]$. 
Traditionally, the metrics most commonly used in exposure assessment have been the arithmetic and geometric mean and standard deviation of an eight-hour time-weighted average $\left(\mathrm{TWA}_{8}\right)$ [5].

Nevertheless, we have to consider that metric appropriateness is dependent on the toxicological mechanism of the chemical studied. For example, in the case of formaldehyde, the health effects are more related with high concentrations than with the exposure time. For that reason, ceilings concentrations might be a better strategy to evaluate exposures and to obtained data to risk assessment development [7].

This study was performed in order to evaluate the importance and influence of environmental monitoring methodology selection in the exposure and in the risk assessment process for formaldehyde occupational exposure.

\section{Materials and methods}

Two environmental monitoring methodologies were simultaneously performed in 10 anatomy and pathology laboratories from Portuguese hospitals, in order to assess formaldehyde occupational exposure. Environmental monitoring was performed between January and March of 2008. Simultaneously, two environmental parameters - temperature and humidity - were monitored, using the Babouc equipment (LSI Sistems), according to the International Standard ISO 7726 - 1998.

In one methodology, environmental samples were obtained by personal air sampling, with low flow pumps, during a typical working day (6 to 8 hours). Formaldehyde levels were measured by GC analysis and the time-weighted average $\left(\mathrm{TWA}_{8}\right)$ estimated according to the NIOSH method (NIOSH 2541) [8]. The sampling was performed near workers nose.

The second methodology aimed to measure ceiling values of formaldehyde using Photo Ionisation Detection (PID) equipment (with $11.7 \mathrm{eV}$ lamp) with simultaneously video recording. Measures were performed in each worker task. This method allows to establish the correlation between worker activities and ceiling values, and also to know the principal exposure sources [9]. Moreover, in this methodology the measures were also performed near the workers nose.

Three exposure groups were defined, namely pathologists, technicians and assistants.

Data obtained from the first methodology was compared with the reference value from Occupational Safety and Health Administration (TLV-TWA=0,75 $\mathrm{ppm}$ ). The ceiling values obtained from the second methodology were compared with the reference value from American Conference of Governmental Industrial Hygienists (TLV $-\mathrm{C}=0,3 \mathrm{ppm})$.

\section{Results}

Table 1 presents the formaldehyde average $\left(\mathrm{TWA}_{8}\right)$ and ceiling concentrations (ppm) obtained in the different laboratories for different exposure groups. 
Table 1: $\quad$ Results of the environmental monitoring methods.

\begin{tabular}{|c|c|c|c|}
\hline Laboratories & Exposure Groups & $\begin{array}{c}\text { Methodology }\left(\mathbf{T W A}_{\mathbf{8}}\right) \\
(\mathrm{ppm})\end{array}$ & $\begin{array}{c}\begin{array}{c}\text { Methodology (Ceiling) } \\
(\mathrm{ppm})\end{array} \\
\end{array}$ \\
\hline \multirow{3}{*}{1} & Assistant & 0,27 & 2,51 \\
\hline & Pathologist & ND & 3,19 \\
\hline & Technicians & 0,16 & 0,938 \\
\hline \multirow{3}{*}{2} & Assistant & 0,15 & 0,62 \\
\hline & Pathologist & 0,24 & 2,71 \\
\hline & Technicians & 0,16 & 3,36 \\
\hline \multirow{3}{*}{3} & Assistant & 0,12 & 0,53 \\
\hline & Pathologist & 0,47 & 2,93 \\
\hline & Technicians & 0,51 & 2,28 \\
\hline \multirow{3}{*}{4} & Assistant & ND & NM \\
\hline & Pathologist & 0,07 & 2,31 \\
\hline & Technicians & 0,11 & 0,85 \\
\hline \multirow{3}{*}{5} & Assistant & ND & NM \\
\hline & Pathologist & 0,06 & 1,10 \\
\hline & Technicians & 0,07 & 0,85 \\
\hline \multirow{3}{*}{6} & Assistant & 0,09 & NM \\
\hline & Pathologist & 0,23 & 0,34 \\
\hline & Technicians & 0,12 & 0,28 \\
\hline \multirow{3}{*}{7} & Assistant & 0,16 & 0,71 \\
\hline & Pathologist & 0,05 & 2,81 \\
\hline & Technicians & 0,04 & 1,26 \\
\hline \multirow{3}{*}{8} & Assistant & 0,25 & 0,68 \\
\hline & Pathologist & 0,11 & 2,08 \\
\hline & Technicians & 0,25 & 0,68 \\
\hline \multirow{3}{*}{9} & Assistant & 0,05 & 0,95 \\
\hline & Pathologist & ND & 0,47 \\
\hline & Technicians & 0,06 & NM \\
\hline \multirow{2}{*}{10} & Pathologist & 0,13 & 5,02 \\
\hline & Technicians & 0,08 & 4,32 \\
\hline
\end{tabular}

ND - not detected.

$\mathrm{NM}$ - not measured.

None of the 29 results of TWA estimation (NIOSH method) were higher than 0,75 ppm (OSHA reference limit-value). Results obtained with PID methodology showed that 25 in 26 measures (96\%) exceeded the Ceiling value in 0,3 ppm (ACGIH reference limit-value). With this second method it was also possible to identify "macroscopy" as the task with the highest formaldehyde exposure levels, and the "pathologists" as the group with the highest ceiling concentrations exposure values $(80 \%)$. 
These results lead to different conclusions about exposure assessment and, consequently, risk assessment.

Concerning temperature, the values laid between 17 and $24^{\circ} \mathrm{C}$ and humidity varied between 33 and $71 \%$. Regarding the influence of environmental variables monitored, no significant correlation $(p>0,05)$ was revealed. Humidity and temperature contributed only in $0,8 \%$ and $2,63 \%$, respectively, to ceiling concentrations variation explanation (Figures 1 and 2).

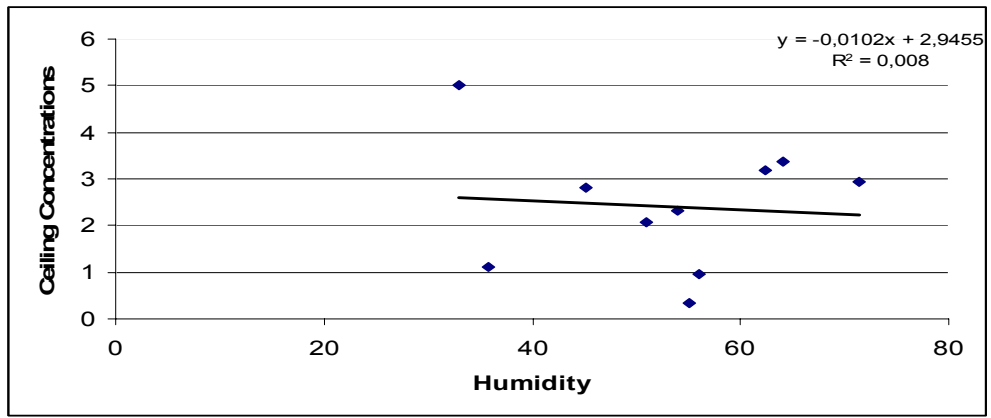

Figure 1: Correlation between humidity and formaldehyde concentrations.

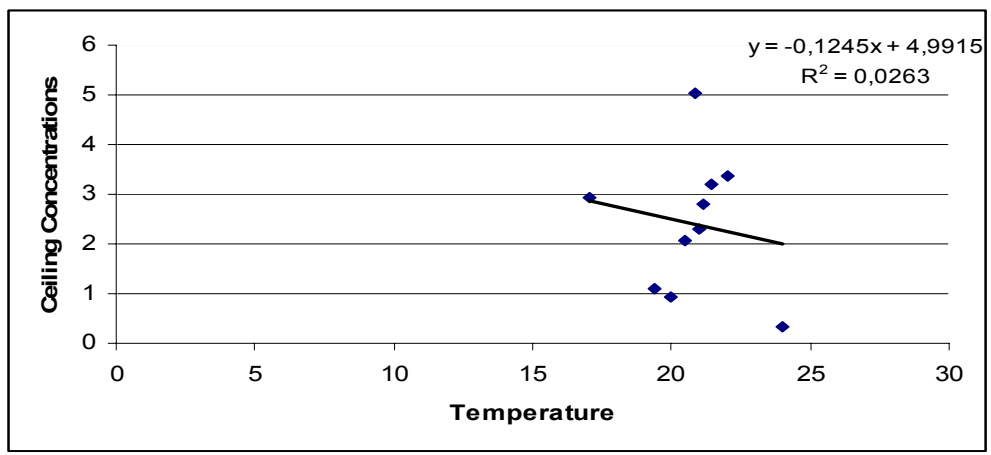

Figure 2: Correlation between temperature and formaldehyde concentrations.

\section{Discussion}

The results obtained are similar to the ones presented in other studies [10-12]. Ceiling values indicated that formaldehyde levels exceed the ACGIH reference $(0,3 \mathrm{ppm})$. It was found that "macroscopy" is the task that involves the highest exposure, probably because precision and visibility are needed, therefore pathologists must lean over the specimen promoting their exposure. Goyer et al. [17] and Orsière et al. [11] studies, also reveal the same kind of results. It is necessary to point out that this type of information (exposure determinants and sources) was only possible because video recording was performed. This 
resource gives the opportunity to directly correlate the performance with exposure $[9,10,13]$.

Measuring TWA $_{8}$ exposures have little usefulness to identify processes that should be targeted to controls since higher concentrations exposure values over short periods are missed by $\mathrm{TWA}_{8}$ methodology [14].

Recommendations from ASHRAE Standard 55 $5^{\text {th }}-1992$ and the Indoor Air Quality, which aim to reduce formaldehyde off-gassing and, consequently, decrease human exposure to this chemical agent, point to $23,5^{\circ} \mathrm{C}$ as the maximum temperature, and for relative humidity values between $30-50 \%$. The values found for each parameter lay above those references.

Results related to environmental variables were not consistent with the expected [15, 16]. There was no statistically significance $(p>0,05)$ in the correlation of formaldehyde ceiling concentrations with temperature and humidity conditions (Figures 1 and 2).

\section{Conclusions}

Due to a tight correlation between health effects (nasopharyngeal cancer) and formaldehyde peak concentrations, selection of the exposure metric should be carefully performed to allow adequate exposure assessment.

Exposure assessment approaches that focus on long-term average exposures, without consideration of the task variability, will fail to give accurate information. To a better exposure control, it is fundamental a correct characterization of the contaminant generation rates associated with specific tasks. This will facilitate effective implementation of controls and work practices.

A reduction of the exposure may be achieved by the use of adequate local exhausts ventilation, relocation of the specimen containers to areas with isolated ventilation and using hooded enclosures over such containers.

Workplace air monitoring is an important tool in airborne concentrations estimation, and can be used to assess the risk level by comparing the measurements with suitable standards. However, in order to accomplish this goal, data must be representative and reliable. It is also important to note that the evaluation of atmospheric exposures is only one of the needed steps to determine the exposure extent.

\section{References}

[1] Greim, H, Snyder, R., Toxicology and risk assessment. A comprehensive introduction. Wiley, 2008.

[2] Ritter, L, Arbuckle, T., Can exposure characterization explain concurrence or discordance between toxicology and epidemiology? Toxicological Sciences. 97 (2007) 241-252.

[3] Herber, R, et al., Risk assessment for occupational exposure to chemicals. A review of current methodology. Pure Applied Chemistry. 73 (2001) 9931031. 
[4] Environmental Protection Agency - Guidelines for exposure assessment. Risk Assessment Forum. U.S. Environmental Protection Agency. Washington DC. (1992).

[5] Stewart, P, Stenzel, M., Exposure assessment in the occupational setting. Applied Occupational and Environmental Hygiene. 15 (2000) 435-444.

[6] Sadhra, S, Gardiner, K., Requirements of monitoring exposure to workplace contaminants. In Sadhra, S, Rampal, K., Occupational Health: risk assessment and management. London: Blackwell Science, 1999. 129158.

[7] IARC - Formaldehyde, 2-Butoxyethanol and 1-tert-Butoxypropan-2-ol. Lyon: International Agency for Research on Cancer, 2006. ISBN 92832 1288 6. (IARC Monographs on the Evaluation of Carcinogenic Risks to Humans; 88).

[8] NIOSH - NIOSH Manual of Analytical Methods, $4^{\text {th }}$ edn. DHEW (NIOSH) Publ. NIOSH, Cincinnati, 1994.

[9] Mcglothlin, J, et al., Occupational exposure assessment and control using video exposure monitoring in the pharmaceutical industry. In International Scientific Conference (IOHA 2005), 6, 19-23 September 2005, Pilanesberg National Park North West Province, South Africa. Pilanesberg: IOHA International Occupational Hygiene Association. SAIOH - Southern African Institute for Occupational Hygiene. MVS - Mine Ventilation Society of South Africa, 2005.

[10] Ryan, T, et al., Video exposure assessments demonstrate excessive laboratory formaldehyde exposures. Applied Occupational and Environmental Hygiene. $18: 6$ (2003) 450-457.

[11] Orsière, T, et al., Genotoxic risk assessment of pathology and anatomy laboratory workers exposed to formaldehyde by use of personal air sampling and analysis of DNA damage in peripheral lymphocytes. Mutation Research. 605 (2006) 30-41;

[12] Ohmichi, K, et al., Formaldehyde exposure in a gross anatomy laboratory. Environmental Sciences and Pollution Research. 13 (2006) 120-124.

[13] Rosén, G, et al., A review of video exposure monitoring as an occupational hygiene tool. Annals of Occupational Hygiene (2005) 1-17.

[14] Susi, P, Schneider, S., Database needs for a task-based exposure assessment model for construction. Applied Occupational Environmental Hygiene. 10 (1995) 394-399.

[15] Arundel, A, et al., Indirect health effects of relative humidity in indoor environments. Environmental Health Perspectives. 65 (1986) 351-361.

[16] Zhang, Y, et al., Influence of temperature on formaldehyde emission parameters of dry building materials. Atmospheric Environment. 41 (2007) 3203-3216.

[17] Goyer, N, et al. - Impacts d'un abaissement de la valeur d'exposition admissible au formaldéhide : Laboratoires de pathologie. Montréal : IRSST - Institut de Recherche Robert-Sauvé en Santé et en Sécurité du Travail, 2004. 